\title{
Neoadjuvant Chemotherapy Consisting of Cisplatin and Nab-paclitaxel Improves the Complete Remission Rate of Locally Advanced Cervical Cancer Patients
}

\author{
Xiao-Li Yu \\ Sun Yat-Sen Memorial Hospital \\ Miao-Fang Wu \\ Sun Yat-Sen Memorial Hospital \\ Lin Ding \\ Sun Yat-Sen Memorial Hospital \\ Jin Yang \\ Sun Yat-Sen Memorial Hospital
}

Shou-Min Bai ( $\sim$ baishm@mail.sysu.edu.cn )

Sun Yat-Sen Memorial Hospital

\section{Research}

Keywords: Neoadjuvant chemotherapy, cervical cancer, complete remission, nab-paclitaxel, chemoradiotherapy

Posted Date: June 23rd, 2021

DOl: https://doi.org/10.21203/rs.3.rs-632036/v1

License: (c) (1) This work is licensed under a Creative Commons Attribution 4.0 International License.

Read Full License 


\section{Abstract}

Background. This study was aimed to determine the effect of neoadjuvant chemotherapy consisting of nab-paclitaxel and cisplatin (NACT-nPC) in patients with locally advanced cervical cancer.

Materials and Methods. The consecutive, newly diagnosed, non-metastatic and locally advanced cervical cancer patients were retrospectively recruited between October 2016 and June 2020 in our hospital. All patients received concurrent chemoradiotherapy alone or following neoadjuvant chemotherapy. Patients were divided into two groups: one receiving NACT-nPC, and the control group receiving no or other regimes of neoadjuvant chemotherapy. We compared the complete remission rate of primary tumor at the end of external radiotherapy and chemoradiotherapy between the two groups.

Results: A total of 198 patients were enrolled in this study, including 60 in NACT-nPC group and 138 in control group. At the end of external radiotherapy, 27 patients in NACT-nPC group and 32 patients in control group achieved complete remission $(P=0.002)$. At the end of chemoradiotherapy, 56 patients in NACT-nPC group and 114 patients in control group achieved complete remission $(P=0.033)$. The acute side effects above grade 3 in NACT-nPC group was $41.7 \%$ (25/60) and manageable, lower than control group which was $77 / 138(55.8 \%)$.

Conclusions. NACT-nPC can improve the complete response rate of patients with locally advanced cervical cancer followed by concurrent chemoradiotherapy, and the toxicity is tolerable. Furthermore, we suspect the NACT-nPC can improve the survival of the patients. However, further prospective studies are needed to confirm this result.

\section{Background}

Cervical cancer ranks the fourth most common and is the sixth leading cause of cancer death in women. Eighty-five percent of cervical cancer patients are located in developing countries [1-2]. Locally advanced cervical cancer (LACC) always involves or exceeds the parametrial tissues, and can not be completely removed through surgery. Concurrent chemoradiotherapy (CCRT) is the main treatment modality [3-6]. However, tumor residual are common after the treatment especially for those with large size, and reduces the tumor control rate and patients' survival. The 5-year overall survival rate of LACC is only $50-75 \%$ [78]. How to reduce the tumor residual, improve the control rate and long-term survival of patients with LACC is the clinical challenge.

Neoadjuvant chemotherapy (NACT) can improve the tumor resection rate and the patients' survival rate. It has been confirmed in many solid tumors such as breast cancer and esophageal cancer [9-10]. However, the role of NACT in cervical cancer is controversial. On the one hand, NACT has been reported to help to implement fertility-sparing surgery for selected patients [11], or improve the resection and survival rate [12]. On the other hand, some literature deemed that the addition of NACT is not superior and is possibly inferior than the CCRT or surgery alone $[8,13]$. Thus, it is important to clarify the role of NACT in cervical cancer. 
The majority of chemotherapy regimens used in the previous literatures are cisplatin combined with traditional paclitaxel or some non-preferred choice $[8,13]$. The albumin bound paclitaxel (nab-paclitaxel) has many advantages over traditional paclitaxel, including higher therapeutic dose, higher distribution in tumor and lower toxicity $[14,15]$. The recommended dose of nab-paclitaxel is almost twice that of traditional paclitaxel. We observed that NACT consisting of nab-paclitaxel and cisplatin (NACT-nPC) obtained the higher tumor response rate than other patients.

Thus, in this study, we intends to retrospectively compare the complete response (CR) rate of the local tumor between patients who underwent NACT-nPC followed by CCRT and the other treatment at the end of external irradiation and CCRT.

\section{Materials And Methods}

\section{Patient characteristics}

Between October 2016 and March 2020, all newly diagnosed, non-metastatic cervical cancer patients, who received radical radiotherapy including external radiation and brachytherapy in our hospital were screened. All clinical records were reviewed by the authors. The inclusion criteria were as follows: (1) The diagnosis as cervical cancer was confirmed with a biopsy; (2) No distant metastasis was confirmed by gynecological and imaging examination; (3) Stage IIA to IIIB; (4) radiation dose was not less than the recommended dose; (5) The pathology was squamous cell carcinoma, adenocarcinoma, or adenosquamous carcinoma. The exclusion criteria were as follows: (1) The pathological types indicating neither adenocarcinoma nor squamous cell carcinoma; (2) Patients received surgery; (3) the presence of other cancers; (4) Pregnant or lactating women; (5) Previous radiotherapy history; (6) The dose of radiotherapy was lower than that of radical treatment, such as giving up halfway; (7) Incomplete clinical treatment data.

\section{The staging}

All the patients received systematic imaging examination including the chest $\mathrm{X}$-ray, abdominal CT scan or ultrasonography, pelvic enhanced MRI or whole body 18 F-FDG PET/CT to exclude distant metastasis. The experienced gynecological oncologists with more than 10 years of clinical experience was consulted to determine the staging of patients. All the patients were staged according to the 2009 International Federation of Gynecology and Obstetrics (FIGO) staging classifications.

\section{The chemotherapy and radiotherapy}

The patients was divided into two groups: the test arm received NACT-nPC, and the control arm received no or other regimens of NACT, such as cisplatin or carboplatin combined with traditional paclitaxel or paclitaxel liposome et al. All patients received concurrent platinum-based chemotherapy. Before 2019, traditional paclitaxel combined with cisplatin was mostly used, while after 2019 , the chemotherapy regimen gradually changed to nab-paclitaxel combined with cisplatin. The nab-paclitaxel and tradition 
paclitaxel was delivered respectively at a dose of $260 \mathrm{mg} / \mathrm{m}^{2}$ and $135 \mathrm{mg} / \mathrm{m}^{2}$, and the cisplatin was $50-$ $75 \mathrm{mg} / \mathrm{m}^{2}$.

All patients received external beam radiotherapy (EBRT) and brachytheray. Intensity Modulated Radiation Therapy was used for EBRT with a total dose of 45-50.4Gy in 25-28 fractions at 1.8Gy/fraction to the planning target volume. Grossly involved nodes were boosted with an additional 10-15Gy of highly conformal (and reduced-volume) EBRT. All patients were treated with one fraction daily over 5 days per week.

At the near end of EBRT, high dose rate brachytherapy was given using an Iridium-192 source. 3-D planning was used in the first three fractions and the remaining was 2-D planning. For 3-D planning, the target volume was contoured on CT according to the interstitial needles and intracavitary applicators. If the vaginal involvement over $1 / 2$, the whole vaginal was included; otherwise, the upper half of vaginal was contoured. The rectum, bladder and sigmoid were delineated as the organs at risk. The prescription was delivered to the outer edge of target volume for 3-D planning and point A for 2-D planning. The brachytherapy was applied with a total dose of 30-36Gy in 5-6 fractions at 6Gy/fraction, 1 fraction daily over 1-2 days per week.

\section{The evaluation of tumor response}

At the end of the EBRT and CCRT, two experienced gynecological oncologists respectively performed a complete gynecological examination to evaluate the tumor situation, and the disagreement was determined through discussion, and the results were recorded in the records. At the end of CCRT, the pelvic ultrasound or enhanced CT/MRI were performed. The treatment response at the end of EBRT was assessed through gynecological examination and the CT scans used in the first 3-D bracheytherapy planning. At the end of CCRT, the treatment response was assessed through gynecological examination and the imaging mentioned above. Complete response was assessed according to RECIST version 1.1 [16].

\section{Follow-up and Statistical analysis}

All patients were assessed every 3 months during the first two years and every 6 months during the next 3 years, and annually thereafter. All patients were followed-up until death or November 15, 2020.

The data were analyzed using SPSS version 21.0. The $\chi^{2}$ test or Fisher's exact test was used to compare ordinal variables and $\mathrm{CR}$ rate between groups. Two-tailed $P$-values $<0.05$ were considered significant.

\section{Results}

\section{Patient characteristics}

Between October 2016 and March 2020, a total of 241 non-metastasis cervical cancer patients received CCRT in our hospital. Forty-three patients were excluded due to the reasons listed in exclusion criteria and 
198 patients were enrolled. Among the 198 included patients, 60 received NACT-nPC and 138 received no or other regimens of NACT.

In all patients, the median age was 55 years. At the time of diagnosis, 134 patients were postmenopausal, and 64 patients were not. The numbers of stage IIA,IIB, IIIAIIIIB were 59, 89, 7 and 43, respectively. 85 patients were imaging-confirmed regional lymph node metastasis and 113 patients had negative lymph node. Ninety-one patients had complications including cardiovascular and cerebrovascular diseases, diabetes, severe liver, kidney or lung diseases. One hundred and seven patients experienced chief complaint symptoms for more than 3 months before treatment. The distribution of squamous cell carcinoma and adenocarcinoma was 181 and 17 patients, respectively, and 142 patients had squamous cell carcinoma antigen higher than the upper limit of normal cutoff. The distribution of the two arms was not significantly different in all the above factors (Table 1 ). 
Table 1

Characteristics of the 198 cervical carcinoma patients enrolled in this study.

\begin{tabular}{|c|c|c|c|}
\hline & $\begin{array}{l}\text { NACT- } n P C^{\&} \\
(n=60)\end{array}$ & $\begin{array}{l}\text { Other regimes } \\
(n=138)\end{array}$ & Pvalue \\
\hline Age (years) & & & 0.083 \\
\hline$\leq 55$ & 25 & 76 & \\
\hline$>55$ & 35 & 62 & \\
\hline menopause & & & 0.262 \\
\hline Yes & 44 & 90 & \\
\hline No & 16 & 48 & \\
\hline Stage (FIGO 2009) & & & 0.290 \\
\hline$\| \mathrm{A}$ & 20 & 39 & \\
\hline IIB & 21 & 68 & \\
\hline IIIA & 3 & 4 & \\
\hline IIIB & 16 & 27 & \\
\hline Regional Lymph node metastasis§ & & & 0.813 \\
\hline Yes & 25 & 60 & \\
\hline No & 35 & 78 & \\
\hline Complication(s) ${ }^{\#}$ & & & 0.625 \\
\hline Yes & 26 & 65 & \\
\hline
\end{tabular}

Abbreviations: NACT-nPC = Neoadjuvant chemotherapy consisting of nab-paclitaxel and cisplatin; SCCA = squamous cell carcinoma antigen; FIGO = International Federation of Gynecology and Obstetrics.

\& Patients received neoadjuvant chemotherapy consisting of nab-paclitaxel and cisplatin followed by concurrent chemoradiotherapy.

* Patients received concurrent chemoradiotherapy alone or following neoadjuvant chemotherapy consisting of traditional paclitaxel and cisplatin or other chemistry agents.

$\S$ Including pelvic lymph node and retroperitoneal lymph node metastasis.

\# Including cardiovascular and cerebrovascular diseases, diabetes, severe liver, kidney and lung diseases.

' $P$-values were calculated using the $\chi^{2}$ test. 


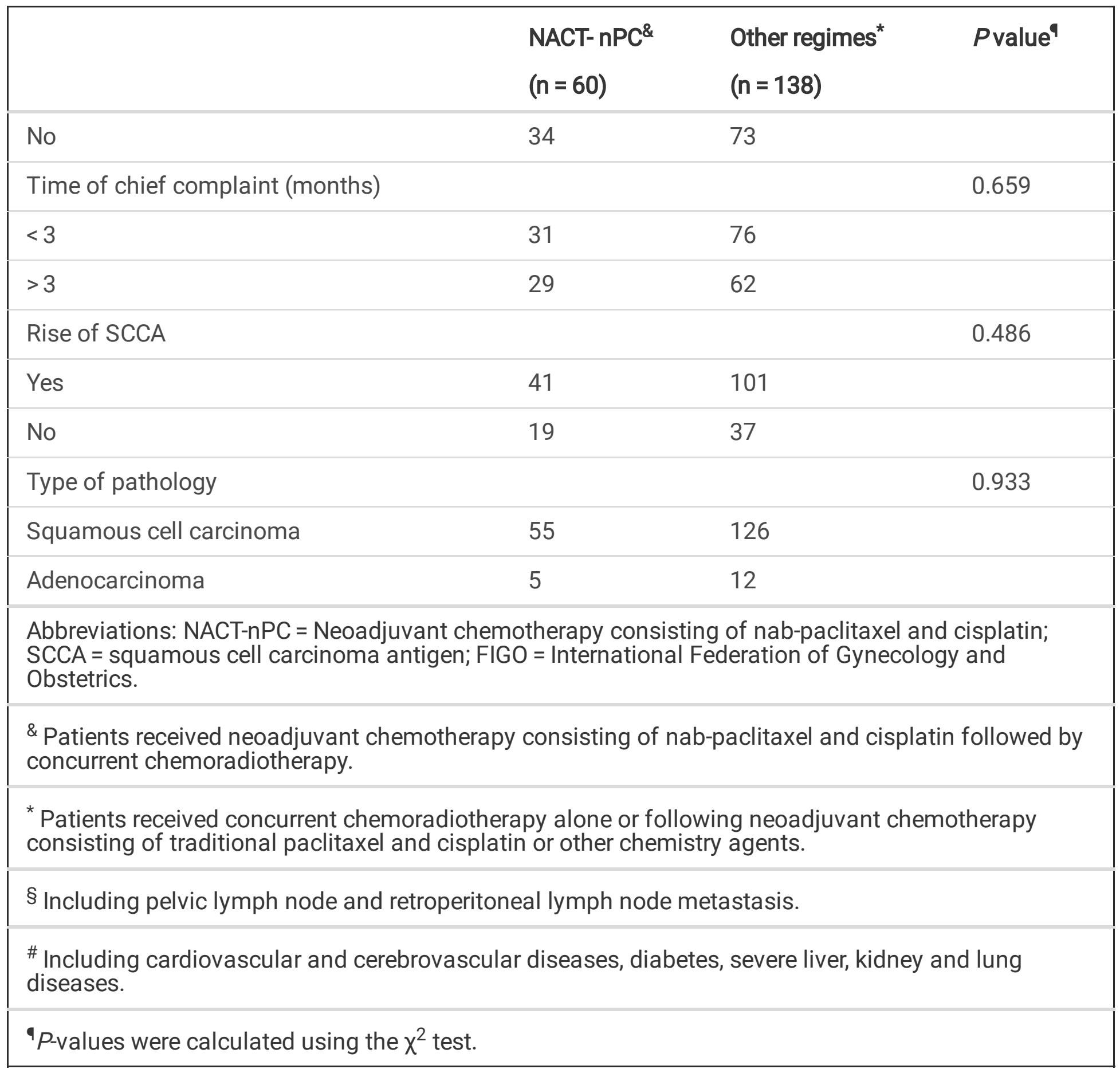

\section{Complete remission rate}

Through the comprehensive assessment, we found that 59 patients achieved $\mathrm{CR}$ at the end of external irradiation, including 27 patients in NACT-nPC group and 32 patients in the control group $(P=0.002)$. At the end of CCRT, 170 patients achieved CR including 56 in the NACT-nPC group and 114 in the control group $(P=0.033)$. The result was shown in Table 2. 
Table 2

The comparison of complete remission after radical chemoradiotherapy between patients with neoadjuvant chemotherapy consisting of cisplatin and albumin-bound paclitaxel and other cervical cancer patients.

\begin{tabular}{|c|c|c|c|}
\hline & $\mathrm{CR}$ & Residual & $P$-value \\
\hline \multicolumn{4}{|l|}{ End of external radiation } \\
\hline NACT- nPC ${ }^{\&}(n=60)$ & 27 & 33 & \multirow[t]{2}{*}{0.002} \\
\hline Other regimes $^{*}(n=138)$ & 32 & 106 & \\
\hline \multicolumn{4}{|l|}{ End of CCRT } \\
\hline NACT- $n P C^{\&}(n=60)$ & 56 & 4 & \multirow[t]{2}{*}{0.033} \\
\hline Other regimes $^{*}(n=138)$ & 114 & 24 & \\
\hline \multicolumn{4}{|c|}{$\begin{array}{l}\text { Abbreviations: NACT-nPC = Neoadjuvant chemotherapy consisting of nab-paclitaxel and cisplatin; } \mathrm{CF} \\
=\text { complete remission; } \mathrm{CCRT}=\text { concurrent chemoradiotherapy. }\end{array}$} \\
\hline \multicolumn{4}{|c|}{$\begin{array}{l}\text { \& Patients received neoadjuvant chemotherapy consisting of nab-paclitaxel and cisplatin followed by } \\
\text { concurrent chemoradiotherapy. }\end{array}$} \\
\hline \multicolumn{4}{|c|}{$\begin{array}{l}\text { * Patients received concurrent chemoradiotherapy alone or following neoadjuvant chemotherapy } \\
\text { consisting of traditional paclitaxel and cisplatin or other chemistry agents. }\end{array}$} \\
\hline
\end{tabular}

\section{Toxicity}

Hematological and gastrointestinal adverse effect were the most common acute toxicity, and the majority of events were manageable. Grade 3-5 hematological toxicity was presented in 100 patients during the whole treatment including 24 in the NACT-nPC group and 76 in the control group. One patient using paclitaxel liposom and carboplatin died nearly after the completion of CCRT for the thrombocytopenia and hemorrhaging. The incidence rate of hematological toxicity above grade 3 in group of NACT-nPC $(24 / 60)$ was lower than the control group $(76 / 138)$ because the preventive use of polyethylene glycol recombinant human granulocyte colony stimulating factor. One patient without hematological toxicity above grade 3 in the NACT-nPC group and control group occured intestinal perforation, respectively, and transferred to intensive care unit and recovered after systematic treatment. Thus, the acute adverse events was $41.7 \%(25 / 60)$ in the NACT-nPC group and $55.8 \%(77 / 138)$ in the control group.

The late adverse events in the group of NACT-nPC including one case of radiation proctitis and one ureteral obstruction. In the control group, one patient occurred colonic fistula and 4 cases of ureteral obstruction. 


\section{Discussion}

Recently, CCRT is still the standard treatment of patients with LACC [3-6], but the tumor control rate and long-term survival are still unsatisfactory, especially for those with stage IIIB or IVA,many of which have tumor residual, progression, even died shortly after CCRT [17]. Therefore, how to reduce the tumor residual and improve their survival is an urgent clinical problem to be resolved.

NACT can reduce the tumor load and lymph-vascular space involvement, increase the radiation sensitivity [18]. For many locally advanced tumors, NACT has been proved to improve the tumor control and survival, and has become the important part of standard treatment [9-10]. However, the role of NACT in cervical cancer is still unclear. A previous meta-analysis of 18 randomized rials found comparing to the radical radiotherapy alone, the addition of NACT can improve the survival for LACC in trials with chemotherapy cycle lengths $\leq 14$ days or cisplatin dose intensities $\geq 25 \mathrm{mg} / \mathrm{m}^{2} /$ week $(\mathrm{HR}=0.83, P=$ 0.046) [19]. In another meta-analysis including early or advanced cervical cancer, the addition of NACT can improve the $\mathrm{OS}(\mathrm{HR}=0.77, P=0.02)$ and progression free survival $(\mathrm{HR}=0.75, P=0.008)$ of patients undergoing radical surgery[20]. Marita et al. retrospectively analyzed the objective response, overall survival and disease specific survival of LACC patients, and found the NACT followed by CCRT results in higher response rates and improvements in disease free survival and disease specific survival compared with the CCRT alone [21]. Conversely, some studies deemed the NACT was detrimental to patients. Da Costa SCS, et al. found compared to the CCRT alone, the addition of NACT consisting of cisplatin and gemcitabine was associated with an inferior 3-year progression free survival ( $40.9 \%$ vs. $60.4 \%, \mathrm{HR}=1.84$; $P=0.033)$ and overall survival $(60.7 \%$ vs. $86.8 \% ; \mathrm{HR}=2.79 ; P=0.006)$, even the lower response rates at the end of treatment $(56.3 \%$ vs. $80.3 \% ; P=0.008)$ [8]. NACT was also reported to be detrimental on survival if cisplatin dose intensities lower than $25 \mathrm{mg} / \mathrm{m}^{2} /$ week $(\mathrm{HR}, 1.35 ; P=0.002)$ or cycle lengths longer than 14 days $(\mathrm{HR}=1.25 ; P=0.005)[19]$.

In light of the reported inconsistent effect of the NACT, it is still of great significance to determine the role and explore the optimal regimens of NACT for LACC. In this study, we found the patients received NACT$\mathrm{nPC}$ followed by CCRT had a higher CR rate than others at the end of EBRT (45.0\% vs. 23.2\%; $P=0.002)$ and CCRT $(93.3 \%$ vs. $82.6 \%, P=0.033)$. At the end of the EBRT, the CR rate of NACT-nPC group was nearly twice that of the control group. We primarily attributed it to the higher therapeutic dose of nab-paclitaxel, whose recommended dose of $260 \mathrm{mg} / \mathrm{m}^{2}$ was nearly twice $135 \mathrm{mg} / \mathrm{m}^{2}$ of traditional paclitaxel, and the advantage of the higher dose translated into a better tumor regression rate. Secondly, the control group included some patients with CCRT alone, who has not completed the CCRT at this time.

After the treatment completion, the CR rate of the control group is $82.6 \%$, similar with that of patients received CCRT alone in previous study [8]. We explain this as follows: 1) The control group in this study included some patients with CCRT alone; 2) NACT regimens in this group included traditional paclitaxel or liposomal paclitaxel combined with cisplatin, carboplatin or other platinum, which have been reported no advantage on survival for LACC [8, 13]; 3) the cisplatin dose intensities in this study was 17-25 $\mathrm{mg} / \mathrm{m}^{2} /$ week, lower than $25 \mathrm{mg} / \mathrm{m}^{2} /$ week, which was the cut-off value of benefit [19]. However, a 
promising clinical CR rate of $93.3 \%$ in group of NACT-nPC was obtained. This result is higher than $56.3 \%$ $-86.54 \%$ reported in previous literature $[8,22]$. Besides the advantage of stronger dose intensities of nabpaclitaxel elaberated above, the following factors may also explain it: 1 ) the NACT reduced the tumor volume, and the massive tumor have more chance to be covered completely with radical radiation dose; 2) NACT reduced the hypoxia of tumor cells and increased its radiation sensitivity [23]; 3) The radiotherapy process from visiting the doctor to CT-simulation, target volume delineation, planning, verification and starting radiation took more than one month on average. During this period, patients with CCRT alone did not receive any treatment, while patients with NACT received chemotherapy usually in one week after the visit and simutaneously start the radiotherapy process. 1-2 cycles of NACT have been performed at the beginning of radiotherapy. Therefore, patients with NACT followed by CCRT received treatment earlier than CCRT alone.

The treatment response to NACT was an independent risk factor of prognosis for patients with LACC [22, 24]. A meta-analysis including 13 studies found the clinical response to NACT was associated with better overall survival $(\mathrm{HR}=3.36, P<0.00001)$ and disease free survival $(\mathrm{HR}=2.36, P<0.00001)$, and the pathological response also predicts favorable overall survival $(H R=5.45, P<0.00001)$ and disease free survival (HR $=3.61, P<0.00001)$ [22]. In another study, $58.9 \%$ of patients with IB2-IIA2 receiving platinumbased NACT achieved response and $8.7 \%$ of patients achieved pathologically CR. NACT responders showed significantly better overall survival $(\mathrm{HR}=2.453, P=0.024)$ and progression free survival $(\mathrm{HR}=$ 2.196, $P<0.013$ ) [24]. We began to use nab-paclitaxel and cisplatin as the NACT from 2019, and the follow-up time was not enough to compare the survival differences between the two treatment schemes. However, compared with the control group, patients receiving NACT-nPC had higher local CR rate at the end of EBRT and CCRT. We speculate that patients receiving NACT-nPC followed by CCRT have better survival outcomes.

In this study, the dosage of nab-paclitaxel was higher than the traditional paclitaxel, which improved the $\mathrm{CR}$ rate of tumor, but did not improve the acute or chronic adverse events. On the contrary, with the preventive use of polyethylene glycol recombinant human granulocyte colony stimulating factor, the hematological toxicity was lower. Besides the hematological toxicity, grade 1-2 diarrhea is also common, which can be relieved after symptomatic treatment. In addition, NACT-nPC did not increase long-term toxicity.

\section{Conclusions}

In conclusion, comparing CCRT, NACT-nPC followed by CCRT can improve the CR rate of patients with LACC at the end of EBRT and CCRT, and the toxicity is tolerable. Furthermore, we suspect the NACT-nPC can improve the long-term survival. However, further prospective studies are needed to confirm this result.

\section{List Of Abbreviations}

NACT-nPC Neoadjuvant chemotherapy consisting of nab-paclitaxel and cisplatin 
LACC Locally advanced cervical cancer

CCRT Concurrent chemoradiotherapy

NACT Neoadjuvant chemotherapy

CR Complete response

FIGO International Federation of Gynecology and Obstetrics

EBRT External beam radiotherapy

\section{Declarations}

\section{Ethics approval and consent to participate}

This retrospective study was approved by the Ethics Committees of Sun Yat-sen Memorial Hospital, Sun Yat-sen University, and the requirement to obtain informed consent was waived. All the data were analyzed anonymously.

\section{Consent for publication}

Not applicable

\section{Availability of data and materials}

The datasets used and/or analysed during the current study are available from the corresponding author on reasonable request.

\section{Competing interests}

The authors indicated no financial disclosures or potential conflicts of interest.

\section{Funding}

Not applicable

\section{Authors' contributions}

Xiao-Li Yu: Investigation; Methodology; Software; Roles/Writing - original draft

Miao-Fang Wu: Project administration; Resources; Supervision

Lin Ding: Formal analysis; Visualization; Supervision

Jin Yang: Data curation; Validation 
Shou-Min Bai: Conceptualization, Funding acquisition; Writing - review \& editing.

\section{Acknowledgements}

Not applicable

\section{References}

1. Siegel RL, Miller KD, Fuchs HE, Jemal A. Cancer Statistics, 2021. CA Cancer J Clin. 2021; 71(1):7-33. https://doi: 10.3322/caac.21654.

2. Ferlay J, Soerjomataram I, Dikshit R, Eser S, Mathers C, Rebelo M, et al. Cancer incidence andmortality worldwide: sources, methods and major patterns in GLOBOCAN2012. Int J Cancer. 2015; 136:E359-86. https://doi.10.1002/ijc.29210.

3. Rose PG, Bundy BN, Watkins EB, Thigpen JT, Deppe G, Maiman MA, et al. Concurrent cisplatin-based radiotherapy and chemotherapy for locally advanced cervical cancer. N Engl J Med. 1999; 340:11441153. https://doi:10.1056/NEJM199904153401502.

4. Morris M, Eifel PJ, Lu J, Grigsby PW, Levenback C, Stevens RE, et al. Pelvic radiation with concurrent chemotherapy compared with pelvic and para-aortic radiation for high-risk cervical cancer. $\mathrm{N}$ Engl J Med. 1999; 340:1137-1143. https://doi: 10.1056/NEJM199904153401501.

5. Lanciano R, Calkins A, Bundy BN, Parham G, 3rd Lucci JA, MooreDH, et al. Randomized comparison of weekly cisplatin or protracted venous infusion of fluorouracil in combination with pelvicradiation in advanced cervix cancer: A gynecologic oncology group study. J Clin Oncol. 2005; 23:82898295. https://doi: 10.1200/JC0.2004.00.0497.

6. Chemoradiotherapy for Cervical Cancer Meta-Analysis Collaboration: Reducing uncertainties about the effects of chemoradiotherapy for cervical cancer: Asystematic review and meta-analysis of individual patient data from 18 randomized trials. J Clin Oncol. 2008; 26:5802-5812. https://doi: 10.1200/JCO.2008.16.4368.

7. Wang YM, Wang CJ, Fang FM, Chen HC, Hsu HCh, Huang YJ, et al. Differences in the outcomes and complications between elderly and younger uterine cervical cancer patients treated by definitive radiotherapy - A propensity score-matched study. Gynecol Oncol. 2017; 145(2):277-283. https://doi: 10.1016/j.ygyno.2017.02.034.

8. da Costa SCS, Bonadio RC, Gabrielli FCG, Aranha AS, Dias Genta MLN, Miranda VC. Neoadjuvant Chemotherapy With Cisplatin and Gemcitabine Followed by Chemoradiation Versus Chemoradiation for Locally Advanced Cervical Cancer: A Randomized Phase II Trial. J Clin Oncol. 2019; 37(33):31243131. https://doi: 10.1200/JC0.19.00674.

9. Mougalian SS, Soulos PR, Killelea BK, Lannin DR, Abu-Khalaf MM, DiGiovanna MP, et al. Use of neoadjuvant chemotherapy for patients with stage I to III breast cancer in the United States. Cancer. 2015; 121(15):2544-52. https://doi: 10.1002/cncr.29348. 
10. Sjoquist KM, Burmeister BH, Smithers BM, Zalcberg JR, Simes RJ, Barbour A, et al. Survival after neoadjuvant chemotherapy or chemoradiotherapy for resectable oesophageal carcinoma: an updated meta-analysis. Lancet Oncol. 2011; 12(7):681-92. https://doi: 10.1016/S14702045(11)70142-5.

11. Tesfai FM, Kroep JR, Gaarenstroom K, Kroon CD, Loenhout RV, Smit V, et al. Fertility-sparing surgery of cervical cancer $>2 \mathrm{~cm}$ (International Federation of Gynecology and Obstetrics 2009 stage IB1-IIA) after neoadjuvant chemotherapy. Int J Gynecol Cancer. 2020; 30(1):115-121. https://doi: 10.1136/ijgc-2019-000647.

12. Fröbe A, Jones G, Bokulić T, Mrčela I, Budanec M, Murgić J, et al. High-dose-rate brachytherapy and concurrent chemoradiotherapy followed by surgery for stage lb-llb cervical cancer: single institution experience. Anticancer Res. 2014; 34(7):3861-6.

13. Gupta S, Maheshwari A, Parab P, Mahantshetty U, Hawaldar R, Chopra SS, et al. Neoadjuvant Chemotherapy Followed by Radical Surgery Versus Concomitant Chemotherapy and Radiotherapy in Patients With Stage IB2, IIA, or IIB Squamous Cervical Cancer: A Randomized Controlled Trial. J Clin Oncol. 2018; 36(16):1548-1555. https://doi: 10.1200/JC0.2017.75.9985.

14. Zhao P, Wang Y, Wu AH, Rao YF, Huang YZ. Roles of Albumin-Binding Proteins in Cancer Progression and Biomimetic Targeted Drug Delivery. Chembiochem. 2018; 19(17):1796-1805. https://doi: 10.1002/cbic.201800201.

15. Chen N, Brachmann C, Liu XP, Pierce DW, Dey J, Kerwin WS, et al. Albumin-bound nanoparticle (nab) paclitaxel exhibits enhanced paclitaxel tissue distribution and tumor penetration. Cancer Chemother Pharmacol. 2015; 76(4):699-712. https://doi: 10.1007/s00280-015-2833-5.

16. Eisenhauer EA, Therasse P, Bogaerts J, Schwartz LH, Sargent D, Ford R, et al. New response evaluation criteria in solid tumours: revised RECIST guideline (version 1.1). Eur J Cancer. 2009; 45(2):228-47. https://doi: 10.1016/j.ejca.2008.10.026.

17. McCormack M, Kadalayil L, Hackshaw A, Hall-Craggs M.A,Symonds RP, Warwick V, et al. A phase II study of weekly neoadjuvant chemotherapy followed by radical chemoradiation for locally advanced cervical cancer. Br J Cancer. 2013; 108(12):2464-9. https://doi: 10.1038/bjc.2013.230.

18. Wang Y, Wang G, Wei LH, Huang LH, Wang JL, Wang SJ. et al. Neoadjuvant chemotherapy for locally advanced cervical cancer reduces surgical risks and lymph-vascular space involvement. Chin J Cancer. 2011; 30(9):645-54. https://doi: 10.5732/cjc.011.10050.

19. Neoadjuvant chemotherapy for locally advanced cervical cancer: a systematic review and metaanalysis of individual patient data from 21 randomised trials. Eur J Cancer. 2003; 39(17):247086. https://doi: 10.1016/s0959-8049(03)00425-8.

20. Rydzewska L, Tierney J, Vale CL, Symonds PR. Neoadjuvant chemotherapy plus surgery versus surgery for cervical cancer. Cochrane Database Syst Rev. 2012; 12(12):CD007406. https://doi: 10.1002/14651858.CD007406.pub3.

21. Marita A, Ordeanu C, Rancea A, Nicolae T, Nagy VM. Long-term survival following neoadjuvant chemotherapy and concomitant radiochemotherapy in locally advanced cervical cancer: results of 
the Oncology Institute "Prof. Dr. Ion Chiricuta" experience. J Med Life. 2018; 11(1):42-50.

22. Zhu YS, Yang JH, Zhang X, Chen DX, Zhang SY. Acquired treatment response from neoadjuvant chemotherapy predicts a favorable prognosis for local advanced cervical cancer: A meta-analysis. Medicine (Baltimore). 2018; 97(17):e0530. https://doi: 10.1097/MD.0000000000010530.

23. Panici PB, Scambia G, Greggi S, Roberto PD, Baiocchi G, Mancuso S. Neoadjuvant chemotherapy and radical surgery in locally advanced cervical carcinoma: a pilot study. Obstet Gynecol. 1988; 71(3 Pt 1):344-8.

24. Huang YH, Liu L, Cai J, Yang L, Sun S, Zhao J, et al. The Efficacy and Response Predictors of Platinum-Based Neoadjuvant Chemotherapy in Locally Advanced Cervical Cancer. Cancer Manag Res. 2020; 12:10469-10477. https://doi: 10.2147/CMAR.S270258. eCollection 2020. 